J. Lake Sci. (湖泊科学) , 2019, 31(6): 1499-1509

DOI 10. 18307/2019. 0620

(c) 2019 by Journal of Lake Sciences

\title{
针改性膨润土针化湖泊中的磷及其生态风险的研究进展”
}

\author{
张巧颖 $^{1,2}$, 杜瑛珣 ${ }^{1 * *}$, 罗春燕 ${ }^{1,2}$, 刘正文 ${ }^{1}$ \\ (1:中国科学院南京地理与湖泊研究所湖泊与环境国家重点实验室,南京 210008) \\ (2: 中国科学院大学, 北京 100049)
}

\begin{abstract}
摘 要: 磷的过度输人是湖泊富营养化的关键原因, 由于内源 (沉积物) 磷的释放, 即使外源磷输人得到控制, 富营养化湖 泊的水质仍难以改善. 近年来, 利用锞改性膨润土 (lanthanum modified bentonite, LMB) 原位钝化沉积物中的磷, 抑制湖泊 内源磷释放备受关注. 为了更好地理解和应用针改性膨润土钝化磷技术, 本文首先介绍锞改性膨润土的组成和其针化磷 的原理, 其次梳理 LMB 磷针化技术在富营养化湖泊中的应用效果, 再分析影响 LMB 钝化磷效率的因素, 最后阐述 LMB 在应用过程中可能产生的生态风险; 并根据以上分析, 提出 LMB 磷针化技术应用中需要注意的方面,对该技术的后续研 究方向进行展望.
\end{abstract}

关键词：锞改性膨润土;富营养化湖泊;沉积物; 磷针化; 生态风险

\section{Advances in researches on phosphorus immobilization by lanthanum modified bentonite in lakes and its ecological risk}

\author{
ZHANG Qiaoying $^{1,2}$, DU Yingxun ${ }^{1 * *}$, LUO Chunyan ${ }^{1,2} \&$ LIU Zhengwen ${ }^{1}$ \\ ( 1: Nanjing Institute of Geography and Limnology, State Key Laboratory of Lake Science and Environment, Chinese Academy \\ of Sciences, Nanjing 210008, P.R. China) \\ (2: University of Chinese Academy of Sciences, Beijing 100049, P.R.China)
}

\begin{abstract}
Phosphorous(P) has been considered a key factor in inducing eutrophication. The internal P loading(mainly P release from the sediment) has been found to delay the lake recovery following the reduction of external loading from the watershed. The control of internal loading by chemical immobilization of $\mathrm{P}$ using the lanthanum( La) modified bentonite( LMB) has been widely applied in recent years. This paper reviews the advances in the immobilization of $\mathrm{P}$ by LMB, including the following aspects: (1) the composition of LMB and mechanism of LMB to immobilize P; (2) the response of P and lake ecosystem to LMB treatment; (3) the factors affecting the treatment efficiency of LMB technology, and(4) the possible ecological risks in the application LMB. After that, the cautions during the LMB application are pointed out and the possible research in this field is proposed.
\end{abstract}

Keywords: Lanthanum modified bentonite; eutrophic lakes; sediment; phosphorus immobilization; ecological risk

磷 (P) 等营养盐的过度输人常常会导致浅水湖泊生态系统从以沉水植物为主的清水态转变为以浮游植 物为主的浑水态 ${ }^{[1-2]}$. 由于内源 (沉积物) 营养盐的释放, 即使外源营养盐负荷得到控制, 富营养化湖泊中湖 水的总磷依然保持较高的浓度 ${ }^{[3-8]}$. 因此, 控制内源磷释放是富营养湖泊修复的关键.

原位化学钝化是国内外用于控制内源磷释放的主要方法之一, 常用的钝化剂包括铁盐 ${ }^{[9]}$ 、铝盐 ${ }^{[10]}$ 和改 性黏土 ${ }^{[11]}$. 近年来, 由于锞改性膨润土 (lanthanum modified bentonite, $\left.\mathrm{LMB}\right)$ 对磷酸根 $\left(\mathrm{PO}_{4}^{3-}\right)$ 具有很强的选 择吸附性,且具有较好的生物相适性等, LMB 在控制内源磷释放中越来越受到关注 ${ }^{[12-13]}$. 据笔者了解, 目前 全面介绍 LMB 磷钝化技术的文献还很有限. 因此, 为了更好地应用 LMB 磷钝化技术, 本文从 LMB 的组成和 钝化磷的机理、LMB 在富营养湖泊中的应用效果、影响 LMB 磷钝化效率的因素和 LMB 磷钝化技术应用的生

* 国家自然科学基金项目 (41671099,41971139) 和中国科学院 STS 项目 (KFJ-STS-ZDTP-038) 联合资助. 2019-01-28 收稿; 2019-06-03 收修改稿. 张巧颖(1994 ) ,女,硕士研究生;E-mail:15806033905@163.com.

** 通信作者;E-mail:yxdu@ niglas.ac.cn. 
态风险四个方面, 阐述 20 多年来 LMB 磷钝化技术的研究进展; 并在此基础上,对 LMB 磷钝化技术的应用和 今后的研究方向提出建议.

\section{LMB 组成与钝化磷的机理}

商业化的 LMB 称为 Phoslock $^{\odot}$ (锁磷剂), 由澳大利亚联邦科学与工业组织 (CSIRO) 于 1990s 开发, 由 $95 \%$ 的膨润土和 $5 \%$ 的稀土锞组成. 膨润土的主要成分为二氧化硅, 结构为两层硅氧四面体的中间夹一层铝 氧八面体, 由共用氧原子将四面体与八面体联结 (图 1a) ; 具有较大的阳离子交换容量 (60 100 meq/100 $\mathrm{g})^{[12]}$. 通过离子交换方法, 可将膨润土层间阳离子置换为镧离子 $\left(\mathrm{La}^{3+}\right)$, 形成钵改性膨润土 (图 $1 \mathrm{~b}$ ). 具体制 备过程为: 将 $0.1 \mathrm{~mol} / \mathrm{L} \mathrm{LaCl}_{3}$ 溶液和高纯度的膨润土以 $100: 1$ (液: 固比) 的比例混合, 摚拌 $24 \mathrm{~h}$ 后在 10000 转/min 下离心; 重复上述步骤 1 遍; 再用蒸馏水洗涤 3 遍, 以去除膨润土上未交换的 $\mathrm{La}^{3+}$; 随后样品于 $60^{\circ} \mathrm{C}$ 下烘干, 密封保存 ${ }^{[15]} . \mathrm{La}^{3+}$ 和 $\mathrm{PO}_{4}^{3-}$ 的结合能力很强 $\left(\mathrm{Ksp}=10^{-24.7} \sim 10^{-25.7} \mathrm{~mol} / \mathrm{L}^{2[16]}\right)$, 在 $\mathrm{pH}$ 值为 $5 \sim 9$ 时能够 以 $1: 1$ 比例反应产生 $\mathrm{LaPO}_{4}^{[17]}$. 存在于膨润土结构中的 $\mathrm{La}^{3+}$, 既保留了与磷酸盐结合的能力, 也不易游离在 水中. 另外, 膨润土具有与沉积物相似的密度和颗粒大小, 沉降后可以作为沉积物的组分从而限制物理再悬 浮或生物扰动 ${ }^{[12]}$, 是一种较为理想的磷钝化剂.

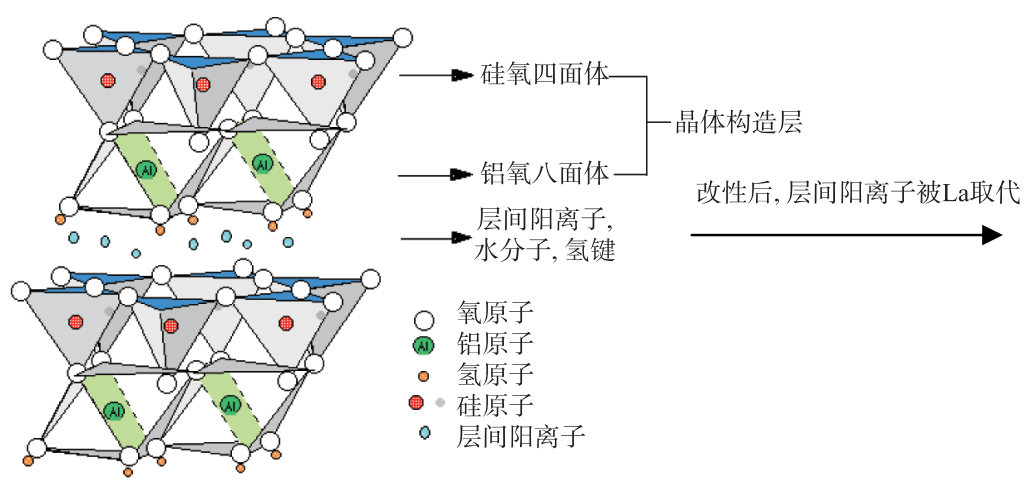

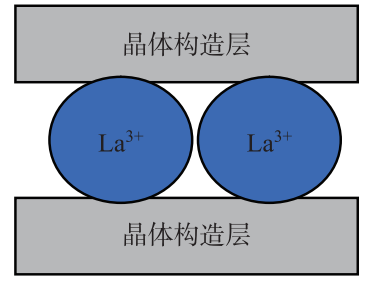

(b)

(a)

图 1 膨润土 (a) 及 $\operatorname{LMB}(\mathrm{b})$ 结构 ${ }^{[12,14]}$

Fig. 1 The structure of bentonite( a) and $\operatorname{LMB}(\mathrm{b})$

实施 LMB 磷钝化技术时, 通常在水体表层或某一深度喷酒 LMB, LMB 在沉降过程中结合水体中的可溶 性活性磷 (SRP), 降低水体中的磷浓度; LMB 沉降后覆盖于沉积物表层形成针化层, 当沉积物中的磷在适当 条件下释放出 $\mathrm{PO}_{4}^{3-}$ 时; 表层的 $\mathrm{LMB}$ 能快速有效捕获 $\mathrm{PO}_{4}^{3-}$, 形成 $\mathrm{LaPO}_{4}$ (图 2). $\mathrm{LaPO}_{4}$ 是一种稳定的化合物, 受环境变化(氧化还原、 $\mathrm{pH}$ 变化等) 影响小, 从而达到抑制沉积物磷释放的作用.

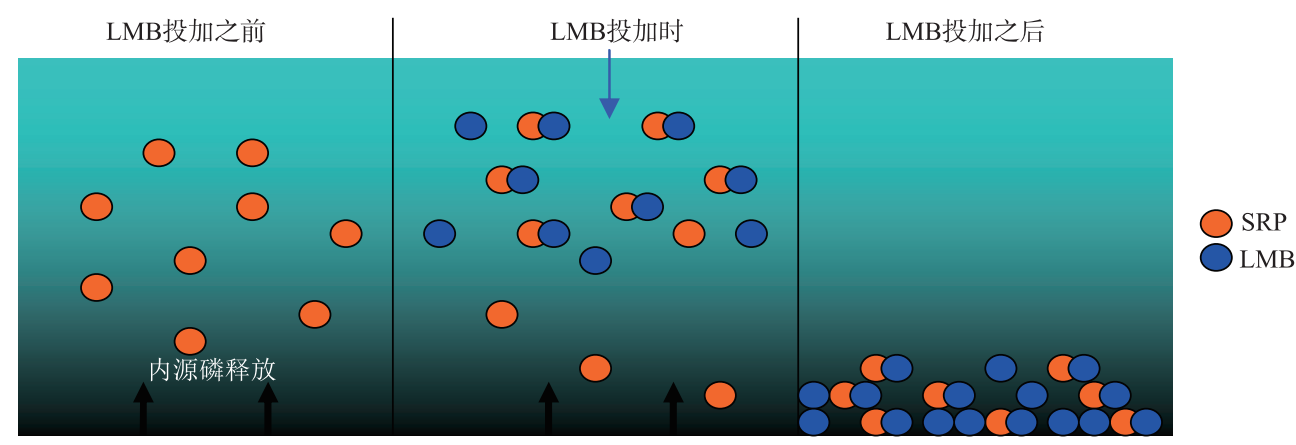

图 2 LMB 磷钝化技术

Fig.2 Phosphorus immobilization by lanthanum modified bentonite 


\section{LMB 磷钝化技术的应用效果}

LMB 磷钝化技术在实际水体中的应用始于 2000 年, 目标水体是澳大利亚西部的 Canning 河 $^{[18]}$, 应用后 水体 FRP (可过滤活性磷) 浓度显著下降. 随后, LMB 在荷兰 ${ }^{[19]}$ 、美国 ${ }^{[20]}$ 、意大利 ${ }^{[21]}$ 、中国 ${ }^{[22]}$ 和墨西哥 ${ }^{[23]}$ 等 国家广泛应用. 迄今为止,LMB 已被应用于 200 多个水体,磷钝化效果明显.

\section{1 降低水柱中的磷浓度}

一方面, LMB 从水体表层向沉积物沉降过程中, 能结合水柱中的 $\mathrm{PO}_{4}^{3-}$, 从而降低水体中的 SRP 浓度; 另 一方面, LMB 钝化层抑制了沉积物中的磷向水柱释放, 进一步降低水体中的磷. 不少研究表明, 经 LMB 磷针 化技术处理后, 水柱中的磷浓度显著降低. Crosa 等 ${ }^{[21]}$ 于 2009-2010 年期间在 Varese 湖进行围隔实验 (围 隔面积 $3.14 \mathrm{~m}^{2}$ ), 投加 $1150 \mathrm{~g} \mathrm{LMB}$ (约 $370 \mathrm{~g} / \mathrm{m}^{2}$ ) 后, 水体磷浓度在短期内大幅降低, 底层水柱中总磷 (TP)、 $\mathrm{SRP}$ 浓度分别从 $110 、 90 \mu \mathrm{g} / \mathrm{L}$ 降至 $40 、 20 \mu \mathrm{g} / \mathrm{L}$, 并在一年时间内维持稳定. 与未处理地点相比, LMB 处理后 底层水中的 TP 和 SRP 浓度降低 $80 \%$ 以上. Spears 等对经 LMB 磷钝化处理 ( LMB 投加量为 $140 \sim 680 \mathrm{~g} / \mathrm{m}^{2}$ ) 24 个月后的 18 个湖泊进行评估 ${ }^{[24]}$, 发现 LMB 处理后, 湖泊的年均 TP 和 SRP 浓度 (18 个湖泊平均值) 均显 著下降: TP 浓度从 $80 \mu \mathrm{g} / \mathrm{L}$ 降至 $30 \mu \mathrm{g} / \mathrm{L}, \mathrm{SRP}$ 浓度由 $19 \mu \mathrm{g} / \mathrm{L}$ 降至 $5 \mu \mathrm{g} / \mathrm{L}$, 下降程度均超过 $60 \%$. 国内学 者采用 LMB 磷针化技术处理富营养化湖泊的研究也表明, LMB 处理能显著降低水柱中的磷浓度: 使用锁磷 剂后, 贵州红枫湖中总磷和磷酸盐浓度减少达 $53.6 \%$ 和 $80.5 \%{ }^{[22]}$. 特别地, 在内源释放严重的夏季, LMB 磷 钝化技术依然有很好的抑制效果: 上述 Spears 评估的 18 个湖泊中,夏季的 TP 和 SRP 浓度仅分别为 40 和 4 $\mu \mathrm{g} / \mathrm{L}^{[24]}$; 利用锁磷剂对杭州西湖沉积物进行磷钝化后, 夏季锁磷剂的控磷效果非常好, 沉积物磷平均释放 通量由 $3.29 \mathrm{mg} /\left(\mathrm{m}^{2} \cdot \mathrm{d}\right)$ 降低到 $0.08 \mathrm{mg} /\left(\mathrm{m}^{2} \cdot \mathrm{d}\right)$, 控制率达 $98 \%{ }^{[25]}$. 这说明 LMB 中的镧能有效捕获夏季因 微生物活动旺盛和缺氧释放的 $\mathrm{PO}_{4}^{3-}, \mathrm{LaPO}_{4}$ 性质稳定, 受环境 (温度升高和氧化还原条件) 变化影响小.

\section{2 钝化沉积物中的磷}

沉积物中磷的赋存形态较为复杂. 沉积物中, 溶解性磷 (间隙水中的) 仅占几个百分点, 绝大多数磷是吸 附或结合在金属 (铁 $(\mathrm{Fe})$ 、铝 $(\mathrm{Al})$ 、锰 $(\mathrm{Mn})$ 和钲 $(\mathrm{Ca})$ 等) 矿物上. 根据欧洲标准测试委员会框架发展起来的 SMT 法 ${ }^{[26]}$, 将沉积物磷形态分为无机磷 (IP) 和有机磷 ( OP) , 其中无机磷包括弱结合态磷 $\left(\mathrm{NH}_{4} \mathrm{Cl}-\mathrm{P}, \mathrm{NH}_{4} \mathrm{Cl}\right.$ 可提取的磷)、碱提取态磷 ( NaOH-P, $\mathrm{NaOH}$ 可提取的磷)、酸提取态磷 ( HCl-P, $\mathrm{HCl}$ 可提取的磷) ; NaOH-P 主 要为与铁/锰/铝的 (氢) 氧化物结合的磷, 通常称为铁/铝磷, HCl-P 为与碳酸钙 (方解石) 结合的或与钙沉淀 形成的钙磷. 其中, 弱结合态磷、铁/铝磷 (还原条件/ $\mathrm{pH}$ 变化后可释放) 和有机磷 (微生物矿化后释放) 被认 为是活性磷 ${ }^{[27]}$, 在一定条件下能转化为 $\mathrm{PO}_{4}^{3-}$ 而释放出来.

由于 $\mathrm{La}^{3+}$ 与 $\mathrm{PO}_{4}^{3-}$ 的结合能力强, 活性磷释放出 $\mathrm{PO}_{4}^{3-}$ 后, 与钝化层中 $\mathrm{La}^{3+}$ 反应形成 $\mathrm{LaPO}_{4} . \mathrm{LaPO}_{4}$ 性质稳 定, 还原条件下不会溶解释放 $\mathrm{PO}_{4}^{3-}$; 在较广的 $\mathrm{pH}$ 范围内 $(5 \sim 9)$ 也不会溶解 ${ }^{[17]}$, 因此可认为是稳定态磷. 在 LMB 磷钝化技术处理后, 观测到沉积物中的磷从活性磷向稳定态磷的转化. Meis 等 ${ }^{[28]}$ 发现 LMB 可以降低 表层沉积物的铁/锰磷, 将其转化为稳定态的磷 ( HCl 可提取的磷). Lin 等 ${ }^{[29]}$ 的实验发现沉积物经过 LMB $\left(510 \mathrm{~g} / \mathrm{m}^{2}\right)$ 处理后, $0 \sim 2 \mathrm{~cm}$ 沉积物的弱结合态磷、铁/镇磷、铝磷和有机磷的含量下降, 而稳定态磷的含量 却大幅度上升. 杨洁等 ${ }^{[30]}$ 的研究还发现, 应用 LMB 进行原位修复后, 底泥中的弱结合态磷、铁磷和铝磷含量 均有不同程度的下降, 分别降低了 $73.0 \%$ 、 $64.0 \%$ 和 $56.1 \%$, 而稳定态的磷含量上升; 由于活性磷含量的下降, 即使在厌氧和碱性的水体 $(\mathrm{pH}=9)$ 中, 沉积物中磷的释放也得到很好的抑制: 杨洁等的研究还发现, 使用 LMB 后, 沉积物磷的释放速率为 $-8.20 \mathrm{mg} /\left(\mathrm{m}^{2} \cdot \mathrm{d}\right)$, 说明 LMB 的应用使沉积物成为磷汇而不是磷源 ${ }^{[30]}$.

\section{3 有利于生态系统从以浮游植物为主的浑水态向以沉水植物为主的清水态的转变}

随着 LMB 对水体中磷的削减和对沉积物磷的钝化, 水体中的 TP 浓度大幅减少, 整体上抑制了浮游植 物的生长; 另外 LMB 的应用改变了水体中 $\mathrm{N} / \mathrm{P}$ 比, 影响了浮游植物群落结构, 总体来讲, 抑制了蓝藻的生 长, 促进了绿藻等的生长. Bishop 等 ${ }^{[20]}$ 在加利福尼亚州 Laguna Niguel 湖用 $413 \mathrm{~g} / \mathrm{m}^{2}$ LMB 处理后, 蓝藻数量 由 $33000 \mathrm{cells} / \mathrm{ml}$ 降至 $1200 \mathrm{cells} / \mathrm{ml}$; 处理前浮游植物群落以蓝藻为主, 处理后藻类种群以绿藻和硅藻为主 (平均为 $6000 \mathrm{cells} / \mathrm{ml}$ ). 相同的, 在 Flemington 湖, Meis 等 ${ }^{[31]}$ 监测了应用 $\operatorname{LMB}\left(170 \mathrm{~g} / \mathrm{m}^{2}\right)$ 前后 30 个月的浮 游植物群落的变化, 发现在第一阶段由于磷浓度的下降导致浮游植物生物量显著下降; 在第二阶段浮游植 
物的相对丰度发生变化, 蓝藻数量显著减少, 而甲藻和绿藻的数量增加.

应用 LMB 修复后, 水体透明度大幅提高, 这可能是 LMB 沉降过程中膨润土对部分悬浮物的吸附, 加上 浮游植物生长的减少导致的; 而透明度的增加促进了沉水植物的生长. 李卫东 ${ }^{[32]}$ 等采用 LMB 对滇池富营养 化水体进行处理, LMB 处理过的实验塘水质透明度从初始的 $0.10 \mathrm{~cm}$ 上升至 $0.84 \mathrm{~cm}$. Spears 等 ${ }^{[24]}$ 对 18 个湖 泊的整理分析也表明,LMB 处理后的湖泊水质透明度增加, 夏天可从 $3.98 \mathrm{~m}$ 增加至 $5.06 \mathrm{~m}$. Jeppesen 等 ${ }^{[33]}$ 对丹麦总磷浓度差异较大的浅水湖泊调查发现, 随着磷浓度降低 $(0.05 \sim 0.10 \mathrm{mg} / \mathrm{L}$ 到 $0 \sim 0.05 \mathrm{mg} / \mathrm{L})$, 水体 透明度从 $1.5 \mathrm{~m}$ 增加到 $3.0 \mathrm{~m}$, 沉水植物物种的数量显著上升 (大约从 8 种上升至 10 种), 最大定植深度可从 $2 \mathrm{~m}$ 增加到约 $6 \mathrm{~m}$. 荷兰的 De Kuil 湖治理前几乎没有水生植物, 应用氯化铁和 LMB 联合处理后的两年, 水生 植物覆盖率明显增加, 几乎达到 $12 \%$; 随着时间的推移, 伊乐藻和普生轮藻成为优势种, De Kuil 湖的大部分 水生植物都是丝状藻 ${ }^{[19]}$.

富营养化湖泊生态修复实践表明, 沉水植物群落恢复是维持湖泊清水态的关键条件 ${ }^{[34]}$. 沉水植物恢复 后, 能通过减少沉积物再悬浮、根系泌氧改善沉积物表层氧化还原条件, 进一步抑制沉积物中的活性磷向上 覆水释放. 根据以上所述 LMB 磷钝化技术的应用结果, 我们认为, 利用 LMB 磷钝化技术能快速降低内源磷 的释放, 改善水质环境, 为沉水植物恢复创造条件; 而沉水植物恢复可减少沉积物 (包括 LMB) 的再悬浮, 进 一步抑制沉积物磷释放, 使系统维持长期的清水态, 两者的联用可能是一种较为理想的富营养湖泊修复 技术.

\section{3 影响 LMB 钝化效果的主要因素}

在 LMB 中, 针化磷的活性成分主要是针; LMB 磷钝化原理可简单概括为 $\mathrm{La}^{3+}$ 和 $\mathrm{PO}_{4}^{3-}$ 的反应. 因此, 影响 $\mathrm{PO}_{4}^{3-}$ 的去除效率的首要因子是与 $\mathrm{PO}_{4}^{3-}$ 反应的有效 $\mathrm{La}^{3+}$ 的量, 由 $\mathrm{LMB}$ 的投加量和水体中可能干扰 $\mathrm{La}^{3+}$ 与 $\mathrm{PO}_{4}^{3-}$ 反应的物质决定. 另外, 风浪和底栖生物扰动可能使钝化层分布不均匀或者厚度减弱, 削弱针化效果.

\section{$3.1 \mathrm{LMB}$ 的投加量}

如果不考虑其他的干扰因素, La 和 P 的反应化学计量比为 $1: 1$; 按照锁磷剂中含 $4.5 \%$ 的 La 计, LMB 的 投加量和所需钝化磷之间的最佳比例为 $100: 1^{[31]}$. 但是如何计算所需钝化的磷是在实际操作过程中需要考 虑的. 正如 2.2 节中所讲, 沉积物中不同形态磷的释放潜力不同, 根据沉积物总磷计算的投加量可能会增加 钝化成本. 因此, 实施 LMB 磷钝化前, 必须明确沉积物中磷的赋存形态. 弱结合态磷和铁磷容易释放进人上 覆水, $\mathrm{OP}$ 也可以通过微生物的矿化产生 $\mathrm{PO}_{4}^{3-}$. 计算需要钝化的磷时, 主要考虑这几种形态的磷. HCl-P 相对 比较稳定, 但也有例外. 有研究表明, 当沉积物中活性钻含量较高时, 在以钙磷为主的沉积物中钙磷的释放 也成为沉积物磷释放的一大来源 ${ }^{[35]}$. 另外, 由于沉积物中的磷和 LMB 都可能发生垂向迁移, 因此, 在计算 LMB 投加量时, 不同形态磷在沉积物中的垂向分布也需要考虑. Guido 等 ${ }^{[19]}$ 发现, 荷兰的 De Kuil 湖采用 LMB 治理后, 随着时间的推移, 沉积物磷释放逐渐增强, 这可能是由于低估了沉积物中潜在可利用磷的量. 治理时 LMB 的投加量是基于沉积物 $0 \sim 5 \mathrm{~cm}$ 的潜在可利用性磷确定的, 但 Guido 等对 De Kuil 湖底部沉积物 磷赋存形态垂向分布的研究表明了在至少 $10 \mathrm{~cm}$ 的沉积物深度存在潜在的生物有效磷.

\section{$3.2 \mathrm{pH}$ 值}

$\mathrm{pH}$ 值可以影响磷酸离子的存在形式及沉积物表面、膨润土表面及活性位点表面所带电荷的特征 ${ }^{[36]}$. 总 的来说, LMB 对 $\mathrm{pH}$ 值 (5 9 范围内) 的依赖性不强, 在中性 $\mathrm{pH}$ 值条件下效果最佳. 在该 $\mathrm{pH}$ 值范围内, 离子 交换与静电吸附是主要的吸附机制. 当 $\mathrm{pH}$ 值在钵氢氧化物等电点 (8.0) 附近时, 磷酸盐与钝化剂表面的 $\mathrm{OH}^{-}$发生离子交换; 当 $\mathrm{pH}$ 值小于等电点时, 膨润土表面的镧活性位点能从水中获取 $\mathrm{H}^{+}$发生质子化, 形成活 性基团 $\equiv \mathrm{La}_{-}-\mathrm{OH}^{2+}$, 通过静电力与磷酸盐发生作用 ${ }^{[37-38]}$. 另外, $\mathrm{H}_{2} \mathrm{PO}_{4}^{-}$是该 $\mathrm{pH}$ 范围内磷酸离子的主要存在形 态, 其吸附自由能是所有磷酸离子中最小的, 因此 $\mathrm{OH}^{2+}$ 对 $\mathrm{H}_{2} \mathrm{PO}_{4}^{-}$的亲和力强, 使得 $\mathrm{LMB}$ 的单位吸附量较 高 ${ }^{[38]}$. 当 $\mathrm{pH}$ 值低于 5 , 钝化剂表面质子化加剧, 离子交换作用减弱, 能吸附的磷酸离子量降低. 当 $\mathrm{pH}$ 值高 于 9 时, 由于 $\mathrm{OH}^{-}$增多, 锚的羟基化增加, 减少了能与 $\mathrm{PO}_{4}^{3-}$ 结合的镧 ${ }^{\left[{ }^{[9]}\right.}$. Mucci 等 ${ }^{[40]}$ 对 $\mathrm{LMB}$ 在不同 $\mathrm{pH}$ 值条 件下 (6 9) 吸附磷能力的研究中发现, 在 $\mathrm{pH}$ 值为 6 时, LMB 吸附 SRP 的效率最高, 吸附量可达 $10.9 \mathrm{mg} / \mathrm{g}$. Ross 等 ${ }^{[41]}$ 通过实验发现, $\mathrm{pH}$ 值在 5 7 时, $\mathrm{LMB}$ 吸附磷酸盐的效率最大, 为 $4.37 \mathrm{mg} / \mathrm{g}$. 当 $\mathrm{pH}$ 值大于 9 时, 吸 
附量降低. Zamparas 等 ${ }^{[42]}$ 比较了 LMB 与未改性膨润土的磷结合效率, 结果表明 LMB 和未改性膨润土均在 $\mathrm{pH}$ 值 6 7 时磷结合效率达到最大, 且 LMB 相较于天然膨润土表现出更低的 $\mathrm{pH}$ 依赖性.

\section{3 水体中的阴离子}

富营养化水体中往往富含其他多种阴离子如 $\mathrm{CO}_{3}^{2-} / \mathrm{HCO}_{3}^{-} 、 \mathrm{Cl}^{-} 、 \mathrm{NO}_{3}^{-}$等, 这些离子会干扰 $\mathrm{La}^{3+}$ 和 $\mathrm{PO}_{4}^{3-}$ 的作 用. $\mathrm{CO}_{3}^{2-} / \mathrm{HCO}_{3}^{-}$能和 $\mathrm{PO}_{4}^{-3}$ 竞争 $\mathrm{La}^{3+}$, 削弱钝化效率. $\mathrm{CO}_{3}^{2-} / \mathrm{HCO}_{3}^{-}$可以与 $\mathrm{La}^{3+}$ 形成 $\mathrm{La}_{2}\left(\mathrm{CO}_{3}\right)_{3}$, 其溶解度 $(\mathrm{Ksp}=$ $\left.3.98 \times 10^{-34} \mathrm{~mol} / \mathrm{L}\right)$ 远低于 $\mathrm{LaPO}_{4}\left(\mathrm{Ksp}=10^{-24.7} \sim 10^{-25.7} \mathrm{~mol} / \mathrm{L}\right)$. 当水体中的 $\mathrm{HCO}_{3}^{-}$浓度较高时, 其与锞活性位 点的吸引力更强, 阻碍 LMB 对磷的吸附, 碱度与 LMB 结合磷的效率之间存在显著的负相关关系 ${ }^{[39,43]} \cdot \mathrm{Cl}^{-}$、 $\mathrm{NO}_{3}^{-}$等阴离子会与 $\mathrm{PO}_{4}^{3-}$ 争夺活性位点, Chen 等 ${ }^{[44]}$ 研究了 $\mathrm{F}^{-} 、 \mathrm{Cl}^{-} 、 \mathrm{SO}_{4}^{2-}$ 和 $\mathrm{NO}_{3}^{-}$对 $\mathrm{La}^{3+}$ 吸附 $\mathrm{PO}_{4}^{3-}$ 的影响, 发现 当 $\mathrm{NO}_{3}^{-}$和 $\mathrm{SO}_{4}^{2-}$ 存在时,磷的去除率从 $89 \%$ 分别下降到 $75.5 \%$ 和 $72.4 \%$; 当 $\mathrm{Cl}^{-}$和 $\mathrm{F}^{-}$存在时, 磷的去除率从 $89 \%$ 分别下降到 $67.3 \%$ 和 $22.8 \%$. Chen 等认为该现象与离子的 $\mathrm{Z} / \mathrm{r}$ (荷电/半径) 有关, $\mathrm{F}^{-}(1 / 1.15)>\mathrm{SO}_{4}^{2-}(2 / 2.40)>$ $\mathrm{Cl}^{-}(1 / 1.81)>\mathrm{NO}_{3}^{-}(1 / 2.81), \mathrm{Z} / \mathrm{r}$ 越大, 干扰越强. 而 $\mathrm{Cl}^{-}$的干扰作用强于 $\mathrm{SO}_{4}^{2-}$, 主要是由于离子交换能力的作 用, $\mathrm{Cl}^{-}$在吸附剂表面替代 $\mathrm{PO}_{4}^{3-}$ 的能力要大于 $\mathrm{SO}_{4}^{2-}$.

\section{4 溶解性有机质}

富营养化的湖泊中大都伴随着较高浓度的溶解性有机质 (DOM) ( 以 DOC 浓度计, 太湖 4 9 mg $\mathrm{C} / \mathrm{L}^{[45]}$, 巢湖 3 10 mg C/L $\mathrm{L}^{[46]}$, , DOM 中存在着羧基和酚差基等官能团, 使 DOM 能与镧发生络合反应, 减少 了与 $\mathrm{PO}_{4}^{3-}$ 结合的镧; 另外, 大分子的 DOM 对膨润土的包裹, 产生位阻效应, 造成 LMB 除磷效率下降. Lürling 等 ${ }^{[47]}$ 通过受控实验研究腐殖质存在情况下锁磷剂对 FRP 的去除率. 短期 $(1 \mathrm{~d})$ 和长期 $(42 \mathrm{~d})$ 的实验都显示 腐殖质抑制了锁磷剂对 FRP 的去除. 在 42 天的实验中, $10 \mathrm{mg} \mathrm{C} / \mathrm{L}$ 的 DOM 存在下 FRP 剩余浓度为 230 $\mu \mathrm{g} / \mathrm{L}$, 而 DOM 不存在时仅 $90 \mu \mathrm{g} / \mathrm{L}$. Yin 等 ${ }^{[48]}$ 的研究证实溶解性有机质通过与金属镧的络合作用减少磷钝 化剂的吸附位点, 使磷钝化剂的吸附能力减弱.

但也有文献称有机质存在下 LMB 与磷的结合能力未必降低, 可能只是结合速率变慢. Dithmer 等 ${ }^{[43]}$ 进 行的长期 (400 天) 磷吸附研究中发现, 腐殖质对 LMB 磷钝化的抑制在 100 天达到最大, 但随着时间的增加, 有机质的抑制作用被削弱, 即 LMB 钝化磷的效果有所增加. Dithmer 等 ${ }^{[43]}$ 认为是有机质与黏土基质相互作 用导致 $\mathrm{LMB}$ 与 $\mathrm{PO}_{4}^{3-}$ 结合速率减慢, 但 $\mathrm{LMB}$ 与磷的结合能力没有降低, 只要反应时间足够, $\mathrm{SRP}$ 将最终与镧 结合, 从而克服有机质的干扰. 但是, Dithmer 等 ${ }^{[43]}$ 在长期实验中采用 $51 \mathrm{mg} / \mathrm{L}$ 的腐殖酸, 未明确说明 400 天 后 DOC 浓度的变化. 由于实验中可能存在微生物作用, 我们推测 400 天后 DOM 抑制作用的削弱也有可能 是有机质的消耗导致.

\section{5 其他因素}

藻类沉淀也会导致磷钝化剂的钝化效率下降, 影响其对富营养化湖泊的控制 ${ }^{[49]}$. 这主要是藻华沉淀导 致的水体厌氧、藻类释放有机物质以及沉积过程中上覆水和沉积物中硫化物的增加. 另外, 藻类中的磷缓慢 释放增加了沉积物中的活性磷, 而磷钝化剂只能作用于相对有限的磷.

风浪可能使钝化层分布不均匀或者厚度减弱, 削弱钝化效果. 水面投加锁磷剂时由于风浪引起的漂移 会影响其分散,干扰锁磷剂的除磷效果. Yasseri 等 ${ }^{\left[{ }^{[} 0\right]}$ 提出将材料直接注人更深的水 (例如 $>2 \mathrm{~m}$ ) 可避免在较 高风速时锁磷剂的不均匀分散. 底栖生物也有可能干扰钝化层, 改变底泥微环境 ${ }^{[51]}$, 但底栖动物对 LMB 钝 化效果的影响还鲜有研究,这方面的研究还有待加强.

\section{LMB 应用的生态风险}

国内外学者已经针对一系列水生生物研究了 La 和 LMB 的毒性效应. Groves 总结了一部分关于 LMB 的 生态毒性效应的研究 ${ }^{[52]}$. LMB 由针和膨润土组成, 在投加初期, 颗粒黏土的增加会使水体变得浑浊; 其次, 渗出的 $\mathrm{La}^{3+}$ 可能会增加水体中锞的浓度; 另外, 沉积物表面钝化层可能改变沉积物性质; 这些因素可能造成 的生态风险值得探讨.

\section{1 微小黏土颗粒对水生生物的影响}

LMB 技术应用的短期内, 微小黏土颗粒对浮游植物、浮游动物和鱼类均可能产生不利影响. 当 LMB 的 浓度高于 $0.5 \mathrm{~g} / \mathrm{L}$ 时, 绿藻和蓝藻的生长受到严重的阻碍, 但 LMB 渗滤液对其生长几乎不产生影响, 且单独 
加人与 LMB 中镧浓度相当的镧时, 影响较 LMB 小的多 ${ }^{[53]}$. 因此推断主要是膨润土颗粒抑制了浮游植物的 生长 ${ }^{[53]}$. 在 Rauwbraken 湖, 使用 PAC ( 聚合氯化铝) 和 LMB 联合处理, 一周后发现浮游动物盔型溞(Daphnia galeata) 消失 ${ }^{[54]}$. 作者排除了 PAC 和 LMB 的急性毒性的原因, 认为可能是由于黏土在短期内使水体浑浊造 成其摄食率的下降. 但是, 这种影响是暂时的, 在几个月后, 盔型掻又重新出现. 悬浮物固体短时间的浓度升 高会影响鱼类的呼吸, 还可能导致幼鱼的死亡, 特别是在卵子发育和鱼苗生长时发生的悬浮固体沉积 ${ }^{[55]}$.

\section{2 镧的溶出及其生态风险}

由于镧离子的溶出,应用 LMB 修复的湖泊可监测到可溶性镧的存在. Spears 等 $^{[56]}$ 观测了 16 个应用 LMB 技术修复的湖泊, 结果显示, 施用 LMB 后, 湖泊表层和底部水的总镧 ( TLa) 和可过滤镧 (FLa) 浓度显著 增加, 由施用前的 $<0.001 \mathrm{mg} / \mathrm{L}$ 到施用后的 $0.026 \sim 2.30$ 和 $0.002 \sim 0.14 \mathrm{mg} / \mathrm{L}$. 不过, 溶出的 $\mathrm{La}^{3+}$ 可能与湖泊 水体中的一些阴离子反应生成沉淀, 从而减少水体中可溶性镧的浓度. 在中高碱度 $(>0.8 \mathrm{mEq} / \mathrm{L})$ 湖泊中, 由 于碱性离子 $\left(\mathrm{OH}^{-}, \mathrm{CO}_{3}^{2-} / \mathrm{HCO}_{3}^{-}\right)$和 $\mathrm{La}^{3+}$ 会产生沉淀反应, $\mathrm{La}^{3+}$ 的浓度非常低 $(<0.0004 \mathrm{mg} / \mathrm{L})$, 但在低碱度湖 泊则有较高浓度的 $\mathrm{La}^{3+}(0.12 \mathrm{mg} / \mathrm{L})^{[56]}$. $\mathrm{PO}_{4}^{3-}$ 的存在也显然能降低可溶性镧的浓度, Martin 和 Hickey 发 现 $^{[57]}$, 当磷的浓度增加 5 倍时, 溶解性针的浓度将降低 3.6 倍.

表 1 总结了 $\mathrm{La}^{3+}$ 和膨润土颗粒对水生生物的影响. 可见, 一定剂量的 $\mathrm{La} / \mathrm{LMB}$ 的渗滤液对浮游动植物及 鱼类的生命活动产生影响. LMB 应用过程中溶出的 $\mathrm{La}^{3+}$ 对水生生物存在一定的威胁.

表 1 LMB 对水生生物的影响

Tab.1 Effects of LMB on aquatic organisms

\begin{tabular}{|c|c|c|}
\hline & $\mathrm{La}^{3+}$ / LMB 渗滤液 & 膨润土颗粒 \\
\hline 水生植物 & $\begin{array}{l}\text { 镧对水鳖 (Hydrocharis dubia) 叶绿体、线粒体和细胞核损害 } \\
\text { 显著, 造成细胞功能紊乱. 其叶绿素的 } \mathrm{EC}_{50} \text { (半最大效应浓 } \\
\text { 度)值为 } 20 \mu \mathrm{mol} / \mathrm{L}^{[58]}\end{array}$ & $\begin{array}{l}\mathrm{LMB} \text { 的浓度高于 } 0.5 \mathrm{~g} / \mathrm{L} \text { 时, 绿藻和蓝 } \\
\text { 藻的生长受到严重的阻碍 } \\
\text { [53] }\end{array}$ \\
\hline 浮游动物 & $\begin{array}{l}\text { 软水中隆线蚤 (Daphnia carinata) 急性实验 }(48 \mathrm{~h}) \mathrm{EC}_{50} \text { 值为 } \\
43 \mu \mathrm{g} / \mathrm{L} \text {, 显著低于硬水中的 } \mathrm{EC}_{50} \text { 值 }(1180 \mu \mathrm{g} / \mathrm{L}) \text {. 慢性实验 } \\
\text { 中, 镧溶度为 } 39 \mu \mathrm{g} / \mathrm{L} \text { 的硬水中, 水溞的死亡率显著 }{ }^{[59]}\end{array}$ & 浊度增加, 导致摄食率下降 ${ }^{[54,63]}$ \\
\hline 鱼类 & $\begin{array}{l}\text { 急性毒性测试中, 彩虹鱼 (Melanotaenia dubouayi) 的 } \mathrm{EC}_{50} \text { 值 } \\
\text { 为 }<600 \mu \mathrm{g} / \mathrm{L}\left(\mathrm{LaCl}_{3}\right), 96 \mathrm{~h} \text { 后存活率为 } 90 \%{ }^{[60]} \text {; 暴露于镧 } \\
\text { 浓度为 } 127 \mu \mathrm{g} / \mathrm{L} \text { 的 } \mathrm{LMB} \text { 渗滤液中, } 96 \mathrm{~h} \text { 后彩虹鱼的存活率 } \\
\text { 为 } 100 \%{ }^{[61]} \\
96 \mathrm{~h} \text { 虹䲡鱼 (Oncorhynchus mykiss) 的 } \mathrm{EC}_{50} \text { 值 ( } \mathrm{LMB} \text { 渗滤液) } \\
\text { 为 } 4350 \mathrm{mg} / \mathrm{L} \text {, 且在 } 48 \mathrm{~h} \text { 时发现死亡, 但是未测得相应的镧 } \\
\text { 浓度 }{ }^{[62]}\end{array}$ & 浊度增加影响鱼类的呼吸 ${ }^{[54,63]}$ \\
\hline
\end{tabular}

溶出的镧是否会影响人类健康是评价 LMB 磷钝化技术生态风险的一个重要方面. LMB 中镧主要可能 通过以下两个途径被人类吸收:一是应用 LMB 修复的水体中存在的可溶性镧通过饮用水直接摄人; 二是镧 通过水生生物富集进而被人类间接摄人. 但是, 从目前的研究结果来看, 通过这两种途径对人类健康造成危 害的可能性很小.

医学上, 碳酸镧常作为治疗高磷血症的一种药剂 ${ }^{[64-66]}$. 镧的口服生物利用性低 $(<0.001 \%)^{[67]}$, 吸收部 分主要由胆汁排出, 少部分由肠道排出体外 ${ }^{[68]}$. 长达 6 年的临床研究未发现碳酸镧对患者肝脏的毒害作 用 ${ }^{[69]}$. 相较于高磷血症患者每日服用的碳酸镧的剂量 $(375 \sim 4500 \mathrm{mg}$ ), 应用 LMB 修复的水体中镧浓度是很 微量的. 因此, 直接饮用 LMB 处理后的水不大可能对人体健康造成直接影响. 另外, 由于水生生物体中镧的 浓度比较小, Landman 等报道的鱼类健康监测报告显示, 虹鯂鱼和小龙虾累积镧的部位主要是肝脏和肝胰腺 组织, 施用 LMB 一个月后, 虹鳟鱼肝脏中检测到镧浓度为 $1.2 \mathrm{mg} / \mathrm{kg}^{[70]}$, 由于含有的镧浓度较低, 加上鱼类 的肝脏通常不被食用,因此, 人类通过食用所摄人的镧不太可能对机体产生伤害.

不过,有必要警惕针暴露可能引起的神经毒性作用: 对大鼠从妊娠的第 0 天到 5 个月口服 $40 \mathrm{mg} / \mathrm{kg}$ 的 镧, 大鼠的记忆力和学习能力显著降低 ${ }^{[71]}$. 同样的, 将小鼠在受孕、妊娠和产后 30 天的饮用水中加人不同浓 
度的针速,小鼠的游泳、行走、触觉、视觉定位等反应显著低于对照组 ${ }^{[72]}$.

\subsection{LMB 覆盖对沉积物理化性质及沉积物中的微生物/底栖生物的影响}

除了膨润土微小颗粒和溶出的 $\mathrm{La}^{3+}$ 可能引起的生态风险, 其他的生态风险包括 LMB 在沉积物表层的覆 盖对沉积物理化性质及沉积物中的微生物/底栖生物的影响. 添加 LMB 后沉积物的理化性质也会发生变 化, 最为突出的是沉积物中溶解氧 (DO) 的变化: 在添加锁磷剂的初期, 由于在沉积物表面形成盖层, 对氧气 的下渗造成一定程度的影响, 因此短期内可能造成沉积物内部溶解氧含量降低, 这与盖层的厚度有一定的 关系. Vopel 等 ${ }^{[73]}$ 研究发现 LMB 会使表层沉积物氧气的下渗深度下降; 与对照组相比 (下渗深度为 $4.4 \pm 0.8$ $\mathrm{mm}$ ), 加人 $200 \mathrm{~g} / \mathrm{m}^{2}$ 的 LMB , $12 \mathrm{~h}$ 后覆盖层一沉积物界面的氧饱和度下降, $\mathrm{O}_{2}$ 下渗深度为 $3.4 \sim 4.2 \mathrm{~mm} ; 400 \mathrm{~g} /$ $\mathrm{m}^{2}$ 的 LMB 剂量 $\mathrm{O}_{2}$ 的下渗深度为 $2.0 \sim 2.2 \mathrm{~mm}$. 另外, 发现较大剂量的 LMB 投加会使沉积物 $\mathrm{pH}$ 值降低 ${ }^{[73]}$.

由于 LMB 可能影响沉积物一水界面的氧气渗透深度、 $\mathrm{pH}$ 值和生物可利用磷浓度, 这些将进一步影响微 生物和底栖生物的活动. Gibbs 等 ${ }^{[74]}$ 发现好氧条件 (曝气) 下 LMB 的应用会在初始时期抑制微生物的硝化 和反硝化作用. 但李卫东等 ${ }^{[32]}$ 的研究指出, LMB 使用一年后滇池水体及底泥中异养微生物和反硝化微生物 量增加了 $30 \% \sim 42 \%$ ( DO 浓度低, $0.36 \sim 0.53 \mathrm{mg} / \mathrm{L}$ ), 有利于 $\mathrm{N}$ 的去除.

\section{5 结论和展望}

由于 $\mathrm{La}^{3+}$ 与磷酸盐有很强的结合能力且生成的磷酸镧性质稳定, LMB 磷钝化技术是控制富营养湖泊沉 积物磷释放的有效方法. 通过沉降水柱中的磷和抑制内源磷释放, LMB 磷针化技术实施后, 水体中的总磷浓 度将会下降, 有利于控制浮游植物的生长、提高水体透明度, 从而促进沉水植物的生长和清水态生态系统的 恢复. 但是, 由于水体和沉积物环境复杂, 水体中普遍存在的溶解性有机质、碳酸根和氢氧根阴离子通过与 $\mathrm{La}^{3+}$ 发生络合反应或沉淀反应而抑制 LMB 磷钝化效率. LMB 的生态风险是其应用过程中必须关注的, LMB 磷钝化技术实施初期, 膨润土微小黏土颗粒和溶出的 $\mathrm{La}^{3+}$ 会对水生生物造成危害, 但随着黏土颗粒的沉降 和溶出的 $\mathrm{La}^{3+}$ 的沉淀, 危害会减弱; 溶出的锢对水生生物可能会产生一定的毒性效应, 不过目前研究显示水 体和水生生物富集的镧浓度均较低, LMB 应用对人类健康产生毒害的可能性较小.

综合 LMB 应用过程中可能出现的问题和风险, 我们对 LMB 磷钝化技术的应用和研究提出以下建议, 希 望为 LMB 磷钝化技术的完善提供一些参考.

1) LMB 实际应用前有必要进行小试和中试: 关注水体环境中共存的各种物质 (影响 LMB 磷针化效率) 、 沉积物磷形态分布 (决定 LMB 投加量的重要因素) 和水生生物对 LMB 投加的响应 (生态风险).

2) 在某些水体中, LMB 技术在应用中存在局限性, 不适合或者不能单独采用 LMB 磷钝化技术. 如藻华 爆发伴随着水体 $\mathrm{pH}$ 值的升高, 当 $\mathrm{pH}$ 值高于 9 时, $\mathrm{LMB}$ 磷钝化技术的效率会降低. 在含盐量较高且碱度较 低的水体中, 较强的离子交换作用使 $\mathrm{La}^{3+}$ 的溶出加剧, 且由于水体碱度低, 溶出的 $\mathrm{La}^{3+}$ 不易沉淀, 造成较强的 生态风险.

3) 探明富营养化湖泊中 DOM 对 LMB 磷钝化技术的影响. DOM 具有多种来源、结构复杂, 目前对 DOM 和 LMB 相互作用研究中, 使用的 DOM 模型物质主要为腐殖酸; 但由于 LMB 技术的应用对象主要为富营养 化湖泊, 因此, 在以后的研究中, 探明藻源性有机质和 LMB 的相互作用及其对 LMB 除磷效率和稳定性的影 响是非常必要的.

4) 加强 LMB 磷针化技术实施对沉积物中生物群落和元素循环影响的研究: 目前对 LMB 覆盖后沉积物 性质的研究多集中在磷形态的变化上, 但磷形态改变后, 活性磷大幅减少, 对沉积物中的生物特别是微生物 会否产生影响, 会否产生磷限制, 这对微生物群落有何影响; 对各种元素如 $\mathrm{C} 、 \mathrm{Fe} 、 \mathrm{~S}$ 的循环的影响也是后续 研究需要考虑的. LMB 磷钝化层和底栖生物之间的相互作用也是后续研究中需要明确的.

5) 关注长时间尺度 LMB 磷钝化技术实施对水生生物的影响研究: 目前大多研究主要针对水生生物的 急性毒性试验, 对于镧在水生生物体内的富集所产生的潜在威胁还未可知, 因此需要评估较高营养级别生 物的潜在毒性. 另外, 虽然目前研究显示镧对人体器官的影响小, 但存在的神经毒性不容忽视, 应加强此方 面的研究.

6) 重视 LMB 磷钝化技术和其他修复技术联用研究,形成高效、稳定的富营养化湖泊修复的集成技术: 
风浪和鱼类、底栖动物的活动在湖泊中不可避免, LMB 实施后, 由于这些因素的影响, 可能会导致 LMB 钝化 层的不稳定, 对钝化技术产生不利的影响. 我们认为 LMB 磷钝化技术和沉水植物恢复技术、食物网 (底栖鱼 类) 控制技术的联用是未来富营养化湖泊修复技术的重要发展方向,这些技术相互促进: 通过控制 LMB 的 实施, 提高了水体的透明度, 有利于沉水植物的生长; 通过沉水植物定植和食物网控制技术, 恢复沉水植物 和减少风浪及底栖生物 (如鱼类) 的干扰, 从而减少 LMB 覆盖层的再悬浮, 增加 LMB 技术的稳定性和长效 性. 后续研究重点是这些技术中关键参数 ( LMB 投加量、沉水植物覆盖率、鱼类密度) 的耦合.

\section{6 参考文献}

[ 1 ] Scheffer M, Carpenter S, Foley JA et al. Catastrophic shifts in ecosystems. Nature, 2001, 413: 591. DOI: 10. $1038 / 35098000$.

[ 2 ] Scheffer M, Carpenter SR. Catastrophic regime shifts in ecosystems: linking theory to observation. Trends in Ecology \& Evolution, 2003, 18(12) : 648-656. DOI: 10.1016/j.tree.2003.09.002.

[ 3 ] Rydin E. Potentially mobile phosphorus in Lake Erken sediment. Water Research, 2000, 34 (7) : 2037-2042. DOI: 10. 1016/S0043-1354(99)00375-9.

[ 4 ] Sondergaard M, Jensen JP, Jeppesen E. Role of sediment and internal loading of phosphorus in shallow lakes. Hydrobiologia, 2003, 506-509(1-3) : 135-145. DOI: 10.1023/B:HYDR.0000008611.12704.dd.

[ 5 ] Qin B, Zhu G, Zhang Let al. Estimation of internal nutrient release in large shallow Lake Taihu, China. Science in Chinese: Series D, 2006, 49(1) : 38-50. DOI: 10.1007/s11430-006-8104-x.

[6] Gong CS, Yao Q, Fan CXet al. Release fluxes estimate of phosphorus in a urban shallow lake: Lake Xuanwu, Nanjing. $J$ Lake Sci, 2006, 18(2) : 179-183. DOI: 10.18307/2006.0212. [ 龚春生, 姚琪, 范成新等. 城市浅水型湖泊底泥释磷 的通量估算——南京玄武湖为例. 湖泊科学, 2006, 18(2): 179-183.]

[ 7 ] Özkundakci D, Hamilton DP, Gibbs MM. Hypolimnetic phosphorus and nitrogen dynamics in a small, eutrophic lake with a seasonally anoxic hypolimnion. Hydrobiologia , 2011, 661 (1) : 5-20. DOI: 10.1007/s10750-010-0358-9.

[ 8 ] Maassen S, Röske I, Uhlmann D. Chemical and microbial composition of sediment in reservoirs with different trophic state. International Review of Hydrobiology, 2003, 88(5) : 508-518. DOI: 10.1002/iroh.200310596.

[ 9 ] Alejandro HC, Carolina DG, Noemí EZ. Effect of operating conditions on the chemical phosphorus removal using ferric chloride by evaluating orthophosphate precipitation and sedimentation of formed precipitates in batch and continuous systems. Chemical Engineering Journal, 2012, 209: 469-477. DOI: 10.1016/j.cej.2012.08.039.

[10] Omoike AI, Vanloon GW. Removal of phosphorus and organic matter removal by alum during wastewater. Water Research, 1999, 33(17) : 3617-3627. DOI: 10.1016/S0043-1354(99)00075-5.

[11] Zou YH, Zhang RY, Chen JA et al. Research advance in the application of clay minerals to phosphorus pollution control in eutrophic water bodies and sediments. Advances in Earth Science, 2018, 33(6) : 578-589. [邹银洪, 张润宇, 陈敬安等. 黏土矿物在富营养化水体和底泥磷污染控制中的应用研究进展. 地球科学进展, 2018, 33(6): 578-589.]

[12] Copetti D, Finsterle K, Marziali L et al. Eutrophication management in surface waters using lanthanum modified bentonite: A review. Water Research, 2016, 97: 162-174. DOI: 10.1016/j.watres.2015.11.056.

[13] Waajen G, van Oosterhout F, Douglas G et al. Geo-engineering experiments in two urban ponds to control eutrophication. Water Research, 2016, 97: 69-82. DOI: 10.1016/j.watres.2015.11.070.

[14] Phoslock ${ }^{\circledR}$. http://www.phoslock.eu.

[15] Douglas. Remediation material and remediation process for sediment. US, 6350383B1. 2002-2-26.

[16] Liu X, Byrne RH. Rare earth and yttrium phosphate solubilities in aqueous solution. Geochemica et Cosmochimica Acta, 1997, 61(8) : 1625-1633. DOI: 10.1016/S0016-7037(97)00037-9.

[17] He SQ, Zhou YY, Lin JW et al. Absorption characteristics of phosphate in water on lanthanum hydroxide-amended sadiments. Environmental Chemistry, 2018, 37(11): 2565-2574. [何思琪, 周亚义, 林建伟等. 氢氧化镧改良沉积物对水 中磷的吸附特征. 环境化学, 2018, 37(11): 2565-2574.]

[18] Robb M, Greenop B, Goss Z et al. Application of Phoslock ${ }^{\mathrm{TM}}$, an innovative phosphorus binding clay, to two Western Australian waterways: Preliminary findings. Hydrobiologia, 2003, 494(1-3) : 237-243. DOI: 10.1023/A: 1025478618611. 
[19] Waajen G, van Oosterhout F, Douglas G et al. Management of eutrophication in Lake De Kuil( The Netherlands) using combined flocculant-Lanthanum modified bentonite treatment. Water Research, 2016, 97: 83-95. DOI: 10. 1016/j. watres.2015.11.034.

[20] Bishop WM, McNabb T, Cormican I et al. Operational evaluation of Phoslock phosphorus locking technology in Laguna Niguel Lake, California. Water, Air and Soil Pollution, 2014, 225(7) : 1-11. DOI: 10.1007/s11270-014-2018-6.

[21] Crosa G, Yasseri S, Nowak KE et al. Recovery of Lake Varese: reducing trophic status through internal P load capping. Fundamental and Applied Limnology, 2013, 183(1) : 49-61. DOI: 10.1127/1863-9135/2013/0427.

[22] Xu EB, Lu D, Du B. Application of innovative Phoslock removal agent in treatment of eutrophic water in the Hongfeng Lake of Guiyang. Environmental Engineering, 2017, 35(supplement 2) : 57-60. [徐恩兵, 路丁, 杜冰. 新型锁磷剂用 于贵阳红枫湖富营养化水体的研究. 环境工程, 2017, 35(增刊 2) : 57-60.]

[23] Márquez-Pacheco H, Hansen AM, Falcón-Rojas A. Phosphorous control in a eutrophied reservoir. Environmental Science and Pollution Research, 2013, 20(12) : 8446-8456. DOI:10.1007/s11356-013-1701-2.

[24] Spears BM, Mackay EB, Yasseri S et al. A meta-analysis of water quality and aquatic macrophyte responses in 18 lakes treated with lanthanum modified bentonite $\left(\right.$ Phoslock $\left.^{\circledR}\right)$. Water Research, 2016, 97: 111-121. DOI: 10.1016/j.watres. 2015.08.020.

[25] Zhu GW, Li J, Zhu MY et al. Efficacy of Phoslock ${ }^{\circledR}$ on the reduction of sediment phosphorus release in West Lake, Hangzhou, China. Environmental Science, 2017, 38(4) : 1451-1459. DOI: 10.13227/j.hjkx.201609123. [ 朱广伟, 李静, 朱 梦园等. 锁磷剂对杭州西湖底泥磷释放的控制效果. 环境科学, 2017, 38(4): 1451-1459.]

[26] Ruban V, Lopez-Sanchez JF, Pardo P et al. Harmonized protocol and certified reference material for the determination of extractable contents of phosphorus in freshwater sediments-A synthesis of recent works. Fresenius Journal of Analytical Chemistry, 2001, 370: 224-228. DOI: 10.1007/s002160100753.

[27] Zhu GW, Qin BQ, Gao G et al. Fractionation of phosphorus in sediments and its relation with soluble phosphorus contents in shallow lakes located in the middle and lower reaches of Changjiang River, China. Acta Scientiae Circumstantiae, 2004, 24(3) : 381-388. DOI: 10.13671/j.hjkxxb.2004.03.003. [朱广伟, 秦伯强, 高光等. 长江中下游浅水湖泊沉积物中 磷的形态及其与水相磷的关系. 环境科学学报, 2004, 24(3) : 381-388.]

[28] Meis S, Spears BM, Maberly SC et al. Assessing the mode of action of Phoslock ${ }^{\circledR}$ in the control of phosphorus release from the bed sediments in a shallow lake(Loch Flemington, UK). Water Research, 2013, 47(13) : 4460-4473. DOI:10.1016/ j.watres.2013.05.017.

[29] Lin JA, Qiu PH, Yan XJ et al. Effectiveness and mode of action of calcium nitrate and Phoslock ${ }^{\circledR}$ in phosphorus control in contaminated sediment, a microcosm study. Water Air and Soil Pollution, 2015, 226: 330. DOI 10.1007/s11270-0152590-4.

[30] Yang J, Liu B, Chang SY et al. Research and application of in situ phosphorus control technology in eutrophic water bodies. Water Resources Protection, 2013, 29(2) : 10-17. [杨洁, 刘波, 常素云等. 富营养化水体原位控磷技术研究 及应用. 水资源保护, 2013, 29(2): 10-17.]

[31] Meis S, Spears BM, Maberly SC et al. Sediment amendment with Phoslock ${ }^{\circledR}$ in Clatto reservoir(Dundee, UK) : Investigating changes in sediment elemental composition and phosphorus fractionation. Journal of Environmental Management, 2012, 93(1) : 185-193. DOI: 10.1016/j.jenvman.2011.09.015.

[32] Li WD, Liu YG, Tian K et al. Study on the application of Phoslock in purifying eutrophic water in Dianchi Lake. Journal of Anhui Agricultural Science, 2010, 38(34) : 19456-19458. [李卫东, 刘云根, 田坤等. Phoslock 应用于滇池富营养 化水体污染净化的研究. 安徽农业科学, 2010, 38(34) : 19456-19458.]

[33] Jeppesen E, Jensen JP, Søndergaard M et al. Trophic structure, species richness and biodiversity in Danish lakes: changes along a phosphorus gradient. Freshwater Biology, 2000, 45(2) : 201-218. DOI: 10.1046/j.1365-2427.2000.00675.x.

[34] Liu Z, Hu J, Zhong P et al. Successful restoration of a tropical shallow eutrophic lake: Strong bottom-up but weak topdown effects recorded. Water Research, 2018, 146: 88-97. DOI: 10.1016/j.watres.2018.09.007.

[35] Jin XD, Wu H, Chen ZM et al. Phosphorus fractions, sorption characteristics and its release in the sediments of Yangtze estuary reservoir, China. Environmental Science, 2015, 36(2): 448-456. DOI: 10.13227/j.hjkX.2015.02.011. [金晓 丹, 吴吴, 陈志明等. 长江河口水库沉积物磷形态、吸附和释放特性. 环境科学, 2015, 36(2) : 448-456.]

[36] Li J. The research of modified clay minerals in the application of in situ coagulation and sedimentation technology [ Disserta- 
tion]. Tianjin: Tianjin University, 2014. [ 李洁. 改性秥土矿物对沉积物磷的原位钝化/掩蔽技术研究 [学位论文]. 天津: 天津大学, 2014.]

[37] Zhang L, Wan LH, Chang $\mathrm{N}$ et al. Removal of phosphate from water by activated carbon fiber loaded with lanthanum oxide. Journal of Hazardous Materials, 2011, 190(1-3) : 848-855. DOI: 10.1016/j.jhazmat.2011.04.021

[38] Haghseresht F, Wang S, Do DD. A novel lanthanum-modified bentonite, Phoslock, for phosphate removal from wastewaters. Applied Clay Science, 2009, 46(4) : 369-375. DOI: 10.1016/j.clay.2009.09.009.

[39] Reitzel K, Andersen FO, Egemose S et al. Phosphate adsorption by lanthanum modified bentonite clay in fresh and brackish water. Water Research, 2013, 47(8) : 2787-2796. DOI: 10.1016/j.watres.2013.02.051.

[40] Mucci M, Maliaka V, Noyma NP et al. Assessment of possible solid-phase phosphate sorbents to mitigate eutrophication: Influence of $\mathrm{pH}$ and anoxia. Science of the Total Environment, 2018, 619/620: 1431-1440. DOI: 10.1016/j. scitotenv. 2017.11.198.

[41] Ross G, Haghseresht F, Cloete TE. The effect of $\mathrm{pH}$ and anoxia on the performance of Phoslock ${ }^{\circledR}$, a phosphorus binding clay. Harmful Algae, 2008, 7(4) : 545-550. DOI: 10.1016/j.hal.2007.12.007.

[42] Zamparas M, Gianni A, Stathi P et al. Removal of phosphate from natural waters using innovative modified bentonites. Applied Clay Science, 2012, 62/63: 101-106. DOI: 10.1016/j.clay.2012.04.020.

[43] Dithmer L, Nielsen UG, Lundberg D et al. Influence of dissolved organic carbon on the efficiency of P sequestration by a lanthanum modified clay. Water Research, 2016, 97: 39-46. DOI: 10.1016/j.watres.2015.07.003.

[44] Chen N, Feng CP, Zhang ZY et al. Preparation and characterization of lanthanum( III) loaded granular ceramic for phosphorus adsorption from aqueous solution. Journal of the Taiwan Institute of Chemical Engineers, 2012, 43: 783-789. DOI: 10.1016/j.jtice.2012.04.003.

[45] Jiang GJ, Ma RH, Duan HT. Estimation of DOC concentrations using CDOM absorption coefficients : a case study in Taihu Lake. Environmental Science, 2012, 33(7) : 2235-2243. DOI: 10.13227/j.hjkx.2012.07.024. [姜广甲, 马荣华, 段洪 涛. 利用 CDOM 吸收系数估算太湖水体表层 DOC 浓度. 环境科学, 2012, 33(7) : 2235-2243.]

[46] Ye LL, Wu XD, Liu B et al. Temporal and spatial distribution characteristics of dissolved organic matter and influencing factors in Lake Chaohu. Environmental Science, 2015, 36(9) : 3186-3193. DOI: 10.13227/j.hjkx.2015.09.009. [叶琳 琳, 吴晓东, 刘波等. 巢湖溶解性有机质时空分布规律及其影响因素. 环境科学, 2015, 36(9) : 3186-3193.]

[47] Lürling M, Waajen G, van Oosterhout F. Humic substances interfere with phosphate removal by lanthanum modified clay in controlling eutrophication. Water Research, 2014, 54(4) : 78-88. DOI: 10.1016/j.watres.2014.01.059.

[48] Yin HB, Du YX, Kong M et al. Interactions of riverine suspended particulate matter with phosphorus inactivation agents across sediment-water interface and the implications for eutrophic lake restoration. Chemical Engineering Journal, 2017, 327 : 150-161. DOI: 10.1016/j.cej.2017.06.099.

[49] Wang CH, Bai LL, Jiang HL et al. Algal bloom sedimentation induces variable control of lake eutrophication by phosphorus inactivating agents. Science of the Total Environment, 2016, 557/558: 479-488. DOI: 10. 1016/j. scitotenv. 2016. 03.082 .

[50] Yasseri S, Epe TS. Analysis of the La:P ratio in lake sediments-Vertical and spatial distribution assessed by a multiplecore survey. Water Research, 2016, 97: 96-100. DOI: 10.1016/j.watres.2015.07.037.

[51] Zhang L, Gu XZ, Fan CX et al. Impact of different benthic animals on phosphorus dynamics across the sediment-water interface. Journal of Environmental Sciences, 2010, 22(11) : 1674-1682. DOI: 10.1016/S1001-0742(09)60305-3.

[52] Groves S. Eco-toxicity Assessment of Phoslock ${ }^{\circledR}$. Phoslock Water Solutions Report Number: TR 02209. PWS, Australia, 2010.

[53] van Oosterhout F, Lürling M. The effect of phosphorus binding clay (Phoslock ${ }^{\circledR}$ ) in mitigating cyanobacterial nuisance: a laboratory study on the effects on water quality variables and plankton. Hydrobiologia, 2013, 710: 265-277. DOI: 10. 1007/s10750-012-1206-x.

[54] van Oosterhout F, Lürling M. Effects of the novel ' flock \& lock' lake restoration technique on Daphnia in Lake Rauwbraken(The Netherlands). Journal of Plankton Research, 2011, 33(2) : 255-263. DOI: 10.1093/plankt/fbq092.

[55] Bilotta GS, Brazier RE. Understanding the influence of suspended solids on water quality and aquatic biota. Water Research, 2008, 42(12) : 2849-2861. DOI: 10.1016/j.watres.2008.03.018.

[56] Spears BM, Lürling M, Yasseri S et al. Lake responses following lanthanum-modified bentonite clay (Phoslock) application: An analysis of water column lanthanum data from 16 case study lakes. Water Research, 2013, 47( 15) : 5930-5942. 
DOI: 10.1016/j.watres.2013.07.016.

[57] Martin ML, Hickey CW. Determination of HSNO ecotoxic thresholds for granular PhoslockTM ( Eureka 1 formulation) phase 1: Acute toxicity. NIWA(National Institute of Water \& Atmospheric Research) Client Report No. HAM2004-137, October 2004.

[58] Xu Q, Fu Y, Min H et al. Laboratory assessment of uptake and toxicity of lanthanum( La) in the leaves of Hydrocharis dubia(Bl.) Backer. Environmental Science and Pollution Research, 2012, 19(9) : 3950-3958. DOI: 10.1007/s11356-0120982-1.

[59] Barry MJ, Meehan BJ. The acute and chronic toxicity of lanthanum to Daphnia carinata. Chemosphere, 2000, 41 (10) : 1669-1674. DOI: 10.1016/S0045-6535(00) 00091-6.

[60] Stauber JL, Binet MT. Canning River Phoslock field trials—Ecotoxicity testing final report. CSIRO Centre for Advanced Analytical Chemistry Energy Techniology. Report no. ET317R. Prepared for CSIRO Land and Water and the WA Water and Rivers Commission. August 2000.

[61] Stauber JL. Toxicity testing of modified clay leachates using freshwater organisms. CSIRO Centre for Advanced Analytical Chemistry Energy Techniology. Report no. ET/IR267R. Prepared for CSIRO Land and Water. March 2000.

[62] Martin ML, Hickey CW. Determination of HSNO ecotoxic thresholds for granular Phoslock ${ }^{\mathrm{TM}}$ (Eureka 1 formulation) phase 1: Acute toxicity. NIWA ( National Institute of Water \& Atmospheric Research) Client Report No. HAM2004-137, October 2004.

[63] Kirk KL. Suspended clay reduces Daphnia feeding rate: behavioral mechanisms. Freshwater Biology, 1991, 25(2) : $357-$ 365. DOI: 10.1111/j.1365-2427.1991.tb00498.x.

[64] Kockx MM, De Meyer GR, Muhring J et al. Apoptosis and related proteins in different stages of human atherosclerotic plaques. Circulation, 1998, 97(23) : 2307-2315. DOI:10.1161/01.CIR.97.23.2307.

[65] Gross P, Six I, Kamel S et al. Vascular toxicity of phosphate in chronic kidney disease: beyond vascular calcification. Circulation Journal Official Journal of the Japanese Circulation Society, 2014, 78(10) : 2339-46. DOI: 10.1253/circj. CJ14-0735.

[66] Jason S, Liu S, Darryl QL. Role of fibroblast growth factor 23 in phosphate homeostasis and pathogenesis of disordered mineral metabolism in chronic kidney disease. Semin Dial, 2007, 20 (4) : 302-308. DOI: 10.1111/j. 1525-139X.2007. 00308.x.

[67] Damment SJ, Pennick M. Clinical pharmacokinetics of the phosphate binder lanthanum carbonate. Clinical Pharmacokinetics , 2008, 47(9) : 553-563. DOI: 10.2165/00003088-200847090-00001.

[68] Pennick M, Dennis K, Damment SJ. Absolute bioavailability and disposition of lanthanum in healthy human subjects administered lanthanum carbonate. Journal of clinical Pharmacology, 2006, 46 ( 7 ): 738-746. DOI: 10. $1177 / 0091270006289846$.

[69] Hutchison AJ, Barnett ME, Krause RJ et al. Lanthanum carbonate study group. Lanthanum carbonate treatment, for up to 6 years, is not associated with adverse effects on the liver in patients with chronic kidney disease stage 5 receiving hemodialysis. Clinical Nephrology, 2009, 71(3) : 286-289. DOI: 10.2215/CJN.03930808.

[70] Landman M, Brijs J, Glover C et al. Lake Okareka and Tikitapu fish health monitoring. Scion, New Zealand: Scion report, 2007.

[71] Feng L, Xiao H, He X et al. Long-term effects of lanthanum intake on the neurobehavioral development of the rat. Neurotoxicology and Teratology, 2006, 28(1) : 119-124. DOI: 10.1016/j.ntt.2005.10.007.

[72] Briner W, Rycek RF, Moellenberndt A et al. Neurodevelopmental effects of lanthanum in mice. Neurotoxicology and Teratology, 2000, 22(4) : 573-581. DOI: 10.1016/S0892-0362(00)00075-1.

[73] Vopel K, Cibbs M, Hickey CW et al. Modification of sediment-water solute exchange by sediment-capping materials: effects on $\mathrm{O}_{2}$ and pH. Marine and Freshwater Research, 2008, 59: 1101-1110. DOI: 10.1071/MF08130.

[74] Gibbs M, Hickey C, Özkundakci D. Sustainability assessment and comparison of efficacy of four P-inactivation agents for managing internal phosphorus loads in lakes: sediment incubations. Hydrobiologia, 2011, 658(1) : 253-275. DOI: 10. 1007/s10750-010-0477-3. 